\title{
Beneficial effects of a soyabean-germ diet on experimental colitis in the ovariectomized rat: involvement of oestrogenic activity
}

\author{
E. Houdeau, L. Chemin, A. Fabre, M. Leveque, L. Bueno, J. Fioramonti, H. Eutamene \\ and V. Theodorou-Bayle \\ UMR 1054 INRA/EI-Purpan, Toulouse, France
}

Soyabean germ (SG), a fraction of soyabean contains a high concentration of bioactive phyto-oestrogens, i.e. isoflavones. It is well established that soyabean isoflavones modulate the activity of the oestrogen receptors (ER) and oestrogen has anti-inflammatory activity in the colon ${ }^{(1)}$. The aim of the present study was to investigate the health potential of dietary SG in relation to bowel inflammation by determining its effects in an animal model of experimental colitis. Here, an investigation of (a) whether dietary SG ameliorates the inflammatory response in rats with colitis and (b) whether this effect involves oestrogenic activity is reported.

Adult female rats were ovariectomized (OVX) and received by oral administration SG ( $0.45 \mathrm{mg}$ isoflavone aglycone equivalents/d in water), $17 \beta$-oestradiol benzoate (EB; $0.6 \mathrm{mg} / \mathrm{kg}$ per $\mathrm{d}$ in maize oil) or vehicle (water or maize oil; controls) daily for $15 \mathrm{~d}$ in the presence or absence of the pure ER antagonist ICI $182780(2 \mathrm{mg} / \mathrm{kg}$ per d subcutaneously from day 10 to day 15). At the end of the treatment period experimental colitis was induced by intra-colonic administration of trinitrobenzene sulfonic acid $(80 \mathrm{mg} / \mathrm{kg})$. After $24 \mathrm{~h} \mathrm{rats}$ were killed and their colons removed for evaluation of the macroscopic damage score (MDS), myeloperoxidase activity (MPO; an index of neutrophil infiltration) and Western blot analysis of macrophage migration inhibitory factor (MIF) expression, an oestrogen-targeted cytokine in inflammatory processes ${ }^{(1)}$.

A marked increase in colonic tissue damage (MDS), MPO activity and MIF expression was observed $24 \mathrm{~h}$ after induction of colitis in comparison with OVX rats without colitis. The vehicles, maize oil or water, did not affect the development of colitis, while EB and SG showed a similar improvement in MDS in the colon (4.7 (SE 0.5) and 5.0 (SE 0.4) for EB and SG groups respectively v. 8.1 (SE 0.4) for the controls; $P<0.001)$. MPO activity was significantly decreased in EB- and SG-treated rats with colitis $(-44 \%$ and $-41 \%$ respectively; $P<0.01)$ when compared with the controls and MIF expression was markedly reduced by SG $(-38 \% ; P<0.05)$. Blockade of ER with ICI 182780 abolished the effects of EB or SG on colitis.

The data show that preventive dietary exposure to SG exerts a protective effect against colonic inflammation in rats that is similar to that of oestrogen treatment. Further, this effect is mediated by ER, suggesting that the anti-inflammatory activity of SG is a result of the oestrogenic activity of isoflavones.

1. Houdeau E, Moriez R, Leveque M, Salvador-Cartier C, Waget A, Leng L, Bueno L, Bucala R \& Fioramonti J (2007) Gastroenterology 132, 982-993. 